\author{
Giovanni Battista La Pegna \\ Filippo Brighina \\ Vincenzo Saporito \\ Antonina Aloisio \\ Calogero Morreale \\ Alfio D'Agati
}

\section{Continuity of healthcare for headache patients: a problem of communication between headache specialists and general practitioners}

Published online: 20 July 2005

\begin{abstract}
The continuous care of headache patients, from headache centres to general practice, is a managerial problem that is still unsolved in Italy. In fact, if on the one hand patients do not usually go to headache centres because of poor information, on the other hand, if they do, they do not find their general practitioner (GP) sufficiently prepared to continue the management. In Sicily we have formed a dense network of headache centres that we will try to link on the Internet to deal with the problem of poor patients information and poor specialist consultation. We also have faced the problem of the continuous care, trying to overcome "the difficulties of comunication between specialists, GPs and patients" and "the difficulties of GPs in diagnostic work", by simple instruments like the Italian version of ID-Migraine, a simple three-item questionnaire.
\end{abstract}

Key words Migraine $\cdot$ Specialist . General practitioner • ID-Migraine • Headache portal $\cdot$ Continuous healthcare

\section{Introduction}

The continuous care of headache patients, from headache centres to general practice, is a managerial problem that is still unsolved in Italy. In fact, if on the one hand patients do not usually go to headache centres [1] because of poor information, on the other hand, if they do, they do not find their general practitioner (GP) sufficiently prepared to continue the management. 
Patients pay the consequences for elements that make continuous assistance difficult: the GP's managerial deficiencies and communication and collaboration problems with specialists who use instruments and a complex language often unintelligible to the GP. For example, diagnostic guidelines that are too specialised and not suitable to GPs' activities and therefore unsuccessfully applied. In fact recent studies indicate that only half of migraine patients receive a correct diagnosis [2]. Consequently we have poor patient care and, especially in southern Italy, an exodus towards northern specialist centres [3].

\section{Method and goals}

In Sicily we have formed, thanks to the SISC, a dense network of headache centres that we will try to link on the Internet to deal with the problem of poor information and poor specialist consultation. We hope to join the network with the principal Italian headache centres by means of the headache portal conceived by Professor Nappi.

We have also been involved in a multicentric work in seven headache centres to improve GPs' diagnostic abilities and to involve them in activities that facilitate patients' continuous care.
The first goal of the work is the validation of the Italian version of ID-Migraine [4], a simple three-item questionnaire that Lipton has recently elaborated to improve migraine identification. The preliminary data have allowed us to confirm that ID-Migraine is a valid screening instrument for the Italian population.

The second goal is the application of ID-Migraine for an epidemiologic study on migraine prevalence in Italy.

The third goal is the evaluation of the application of ID-Migraine in general practice, suitably studied for GPs instead of the complex international guidelines.

\section{Discussion}

The GPs' diagnostic difficulties and the communication problems between GPs and specialists can be overcome if the specialists refrain from teaching complex instruments in favour of easier ones.

We hope this work will induce GPs to use IDMigraine; it will allow them to learn a new and simple diagnostic instrument and a language with which they can communicate easily with specialists.

All these will guarantee, according to us, a better and lasting dedication of GPs to the work of headache centres, assuring, in this way, continuous healthcare for headache patients.

\section{References}

1. The Headache Week (2005) A meeting between patients and experts: forward to 19th National Congress of the Italian Society for Study of Headache. Venice, 29 September-2 October

2. Lipton RB, Scher AI, Kolodner K, Liberman J, Steiner TJ, Stewart WF (2002) Migraine in the United States: epidemiology and patterns of health care use. Neurology 58:885-894
3. Iannacchero R, Cannistrà U, Trinchi RL, Corasaniti F, Grillo T, De Caro E (2002) Studio epidemiologico trasversale sulle sindromi emicraniche in un campione di popolazione della Calabria. XVI Congresso Nazionale SISC, Chieti, p 85
4. Lipton RB, Dodick D, Sadovsky R, Kolodner K, Endicott J, Hettiarachchi J, Harrison W; ID Migraine validation study (2003) A self-administered screener for migraine in primary care: the ID Migraine validation study. Neurology 61:375-382 